\title{
Modeling and Simulation of Voltage Frequency Ratio Control System for Asynchronous Motor
}

\author{
Jun Rong, Yuejiao Ding, Xi Chen, Li Wan \\ Department of Information and Communication, \\ Hunan Institute of Science and Technology. \\ Yueyang, China 414006 \\ e-mail:rj1219@163.com
}

\begin{abstract}
Alternating current (AC) machine speed regulation system plays a very important role in our industrial and agricultural production. This paper focuses on the three-phase asynchronous motor which account for most of the proportion of the AC machines. Firstly, this paper establishes three mathematical models of asynchronous motor based on dq coordinate system and introduces the principle of V/f speedadjusting for asynchronous motor, and then the paper builds the simulation models of V/f speed-adjusting control system for asynchronous motor based on Matlab/Simulink. The simulation results of the $V / f$ speed-adjusting control system verifies the correctness of the simulation models and the principle of speed regulation.
\end{abstract}

Keywords- asynchronous motor; V/f control; mathematical model; dq coordinate system; simulation;

\section{INTRODUCTION}

DC speed-adjusting system has the advantages of good starting and braking performance, wide range of speedadjusting, the little static error and stability, and its theory and practice have been relatively mature and application. But mechanical reversing of DC motor restricts the main technology index of capacity, the maximum voltage, maximum speed and overload capacity, and also limits the development of DC speed-adjusting system, so the study of $\mathrm{AC}$ motor to replace $\mathrm{DC}$ motor is to be imperative, and now AC adjustable speed research and application has been obtained great development ${ }^{[1][2]}$. The main advantage for $\mathrm{AC}$ speed-adjusting system have no brush and commutator, and the $\mathrm{AC}$ motor has simple structure, reliable operation, long service life, easy maintenance, and also the price is lower than the equivalent capacity of DC motor.

\section{THE MATHEMATICAL MODEL OF THREE-PHASE ASYNCHRONOUS MOTOR IN DQ COORDINATE SYSTEM}

The mathematical model of asynchronous motor based on $A B C$ coordinate system contains time varying inductance matrix, and every volume is coupled each other. So it is dealt very complex, and it is used to simplify the mathematical model with the coordinate transformation. Three-phase static $A B C$ coordinates on a mathematical model for the complex is an important factor in the windings without decoupling. If it is transformed to the two-phase $(d q)$ coordinate system, and the motor will be simplified because the two-phase coordinate system is perpendicular to each other, and the two-phase has no magnetic coupling between windings, and the mathematical

\author{
Yiming $\mathrm{Li}$ \\ Department of Computer, \\ Hunan Institute of Science and Technology. \\ Yueyang, China 414006 \\ e-mail:93210179@qq.com
}

system is perpendicular to each other, and the two-phase has no magnetic coupling between windings, and the mathematical

model is also simple. Now builds the mathematical model in two-phase coordinate system.If the mathematical model of motor is transformed from three-phase static coordinate system to the $d q$ coordinate system, the voltage, current, flux and torque of the stator and the rotor should be transformed into the $d q$ coordinate system by use of $3 / 2$ transformation, then transforms the variables into the $d q$ coordinate system by use of the rotation matrix $2 s / 2 r$. The physical model in $d q$ coordinate system is follow as Figure1.

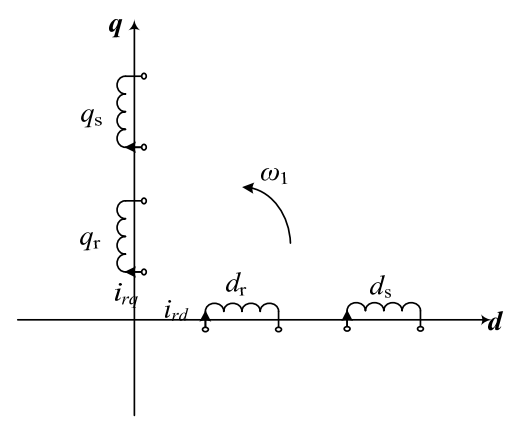

Figure1. The physical model of asynchronous motor in $d q$ coordinate system

The key that builds mathematical model of asynchronous motor in $d q$ coordinate system is to get transformation matrix of three-phase static coordinate system to two-phase rotation coordinate system ${ }^{[3][4]}$. So it needs the experience of $3 / 2$ transform, $2 s / 2 r$ transform, and the transformation matrix is shown as type(1).

$$
\boldsymbol{C}_{3 \mathrm{~s} / 2 \mathrm{r}}=\boldsymbol{C}_{2 \mathrm{~s} / 2 \mathrm{r}} \boldsymbol{C}_{3 / 2}=\sqrt{\frac{2}{3}}\left[\begin{array}{ccc}
\cos \vartheta & \cos \left(\theta-120^{\circ}\right) & \cos \left(\theta+120^{\circ}\right) \\
-\sin \theta & -\sin \left(\theta-120^{\circ}\right) & -\sin \left(\theta+120^{\circ}\right) \\
\frac{1}{\sqrt{2}} & \frac{1}{\sqrt{2}} & \frac{1}{\sqrt{2}}
\end{array}\right]
$$

The inverse matrix is shown as type(2).

$$
\boldsymbol{C}_{2 \mathrm{r} / 3 \mathrm{~s}}=\boldsymbol{C}_{3 \mathrm{~s} / 2 \mathrm{r}}^{-1}=\boldsymbol{C}_{3 \mathrm{~s} / 2 \mathrm{r}}^{T}=\sqrt{\frac{2}{3}}\left[\begin{array}{ccc}
\cos \vartheta & -\sin \theta & \frac{1}{\sqrt{2}} \\
\cos \left(\theta-120^{\circ}\right) & -\sin \left(\theta-120^{\circ}\right) & \frac{1}{\sqrt{2}} \\
\cos \left(\theta+120^{\circ}\right) & -\sin \left(\theta-120^{\circ}\right) & \frac{1}{\sqrt{2}}
\end{array}\right]
$$


The matrix is power invariant transformation for $\boldsymbol{C}_{2 \mathrm{r} / 3 \mathrm{~s}}=\boldsymbol{C}_{3 \mathrm{~s} / 2 \mathrm{r}}^{-1}=\boldsymbol{C}_{3 \mathrm{~s} / 2 \mathrm{r}}^{T}$, and it can get the mathematical model of asynchronous motor in $d q$ coordinate system according to $3 s / 2 r$ transformation matrix and its inverse matrix for dynamic mathematical model. The flux equation is shown as type(3).

$$
\left[\begin{array}{l}
\psi_{\mathrm{sd}} \\
\psi_{\mathrm{sq}} \\
\psi_{\mathrm{rd}} \\
\psi_{\mathrm{rq}}
\end{array}\right]=\left[\begin{array}{cccc}
L_{\mathrm{s}} & 0 & L_{\mathrm{m}} & 0 \\
0 & L_{\mathrm{s}} & 0 & L_{\mathrm{m}} \\
L_{\mathrm{m}} & 0 & L_{\mathrm{r}} & 0 \\
0 & L_{\mathrm{m}} & 0 & L_{\mathrm{r}}
\end{array}\right]\left[\begin{array}{l}
i_{\mathrm{sd}} \\
i_{\mathrm{sq}} \\
i_{\mathrm{rd}} \\
i_{\mathrm{rq}}
\end{array}\right]
$$

$L_{\mathrm{m}}=\frac{3}{2} L_{\mathrm{ms}}-$ The inductance of equivalent two-phase stator windings in $d q$ coordinate system

$$
L_{\mathrm{r}}=\frac{3}{2} L_{\mathrm{ms}}+L_{\mathrm{lr}}=L_{\mathrm{m}}+L_{\mathrm{r} \sigma} \quad \text { The mutual }
$$

inductance of coaxial equivalent winding for stator and rotor windings in $d q$ coordinate system

$$
L_{\mathrm{s}}=\frac{3}{2} L_{\mathrm{ms}}+L_{\mathrm{ls}}=L_{\mathrm{m}}+L_{\mathrm{s} \sigma}-\text { The inductance of }
$$

rotor equivalent two-phase windings in $d q$ coordinate system.

The voltage equation is shown as type(4).

$$
\left.\begin{array}{l}
u_{\mathrm{sd}}=R_{\mathrm{s}} i_{\mathrm{sd}}+p \psi_{\mathrm{sd}}-\omega_{\mathrm{dqs}} \psi_{\mathrm{sq}} \\
u_{\mathrm{sq}}=R_{\mathrm{s}} i_{\mathrm{sq}}+p \psi_{\mathrm{sq}}+\omega_{\mathrm{dqs}} \psi_{\mathrm{sd}} \\
u_{\mathrm{rd}}=R_{\mathrm{r}} i_{\mathrm{rd}}+p \psi_{\mathrm{rd}}-\omega_{\mathrm{dqr}} \psi_{\mathrm{rq}} \\
u_{\mathrm{rq}}=R_{\mathrm{r}} i_{\mathrm{rq}}+p \psi_{\mathrm{rq}}+\omega_{\mathrm{dqr}} \psi_{\mathrm{rd}}
\end{array}\right\}
$$

According to the type(3) and type(4), it can get the type as follow as type(5).

$$
\begin{aligned}
{\left[\begin{array}{l}
u_{\mathrm{sd}} \\
u_{\mathrm{sq}} \\
u_{\mathrm{rd}} \\
u_{\mathrm{rq}}
\end{array}\right]=\left[\begin{array}{cccc}
R_{\mathrm{s}} & 0 & 0 & 0 \\
0 & R_{\mathrm{s}} & 0 & 0 \\
0 & 0 & R_{\mathrm{r}} & 0 \\
0 & 0 & 0 & R_{\mathrm{r}}
\end{array}\right]\left[\begin{array}{l}
i_{\mathrm{sd}} \\
i_{\mathrm{sq}} \\
i_{\mathrm{rd}} \\
i_{\mathrm{rq}}
\end{array}\right]+\left[\begin{array}{cccc}
L_{\mathrm{s}} p & 0 & L_{\mathrm{m}} p & 0 \\
0 & L_{\mathrm{s}} p & 0 & L_{\mathrm{m}} p \\
L_{\mathrm{m}} p & 0 & L_{\mathrm{r}} p & 0 \\
0 & L_{\mathrm{m}} p & 0 & L_{\mathrm{r}} p
\end{array}\right]\left[\begin{array}{l}
i_{\mathrm{sd}} \\
i_{\mathrm{sq}} \\
i_{\mathrm{rd}} \\
i_{\mathrm{rq}}
\end{array}\right] } \\
+\left[\begin{array}{cccc}
0 & -\omega_{\mathrm{dqs}} & 0 & 0 \\
\omega_{\mathrm{dqs}} & 0 & 0 & 0 \\
0 & 0 & 0 & -\omega_{\mathrm{dqr}} \\
0 & 0 & \omega_{\mathrm{dqr}} & 0
\end{array}\right]\left[\begin{array}{l}
\psi_{\mathrm{sd}} \\
\psi_{\mathrm{sq}} \\
\psi_{\mathrm{rd}} \\
\psi_{\mathrm{rq}}
\end{array}\right]
\end{aligned}
$$

Type(5) is a nonlinear dynamic voltage equation of asynchronous motor. In type(5), $R$ represents the resistance drop, $L p$ represents the inductive drop, and $w$ is rotating electromotive force. The matrix of $L$ is $4 \times 4$ constant coefficient linear matrix.

The torque equation is shown as type(6).

$$
T_{\mathrm{e}}=n_{\mathrm{p}} L_{\mathrm{m}}\left(i_{\mathrm{sq}} i_{\mathrm{rd}}-i_{\mathrm{sd}} i_{\mathrm{rq}}\right)
$$

The motion equations is shown as type(7).

$$
T_{\mathrm{e}}=T_{\mathrm{L}}+\frac{J}{n_{\mathrm{p}}} \frac{d \omega}{d t}
$$

So the dynamic equivalent circuit diagram of asynchronous motor is shown as Figure2:

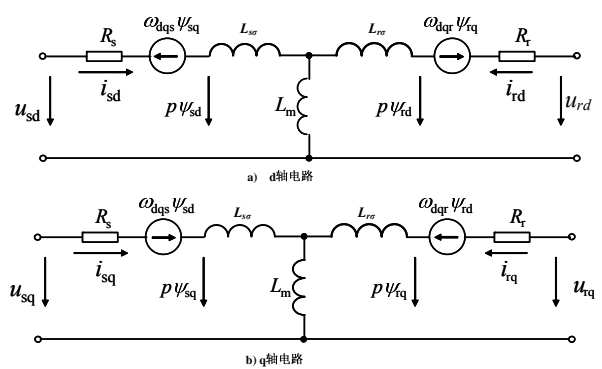

Figure2. The dynamic equivalent circuit of asynchronous motor in $d q$ coordinate system

\section{THE SPEED-ADJUSTING THEORY OF VOLTAGE} FREQUENCY RATIO CONTROL FOR ASYNCHRONOUS MOTOR

Scalar control system is to control a magnetmomotive force amplitude and rotating speed in chart3, and do not control the angle between them. The reason is that the amplitude and speed of rotation are scalar, hence the scalar control system of voltage to frequency ration $\operatorname{control}(V / f)$ is base on the steady state relationship between the control of open-loop system, and has no torque control loop, so the system dynamic performance isn't good. But this kind of control mode of the inverter has the advantages of simple structure, low cost, reliable operation, convenient speed regulation, and it is widely applied to the dynamic performance requirements of high speed, small occasions, wind machine, water pump. The premise of motor speed-adjusting control is to make full use of iron core and the wire of the motor, and remains the core flux density and the current density of wires stable. The effective value of phase electromotive force for three-phase asynchronous motor is shown as type(8).

$$
E_{s}=\sqrt{2} \pi f_{1} N_{s} k_{N s} \Phi_{m}
$$

If make flux keep rated value unchanged, the electromotive force and frequency ratio should be kept unchanged.

$$
\frac{E_{s}}{f_{1}}=\text { const }=\frac{E_{s N}}{f_{1 N}}
$$

The rated value of flux is rated magnetomotive force, and the constant value is $\frac{E_{s N}}{f_{1 N}}$. It is known as constant voltage frequency ratio control method because the magnetomotive force magnetic force and frequency ration is constant value. $V / f$ can be discussed by steady-state equivalent circuit because $V / f$ is the control mode of steady-state relationship ${ }^{[5]}$. 


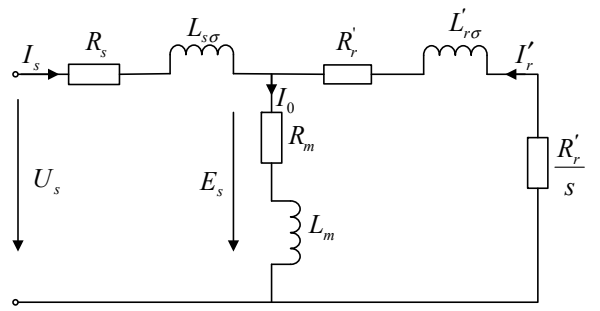

Figure3. The steady-state equivalent circuit of asynchronous motor

It can be got the type (10)from the formula of electromagnetic moment.

$$
T_{e}=\frac{P_{e m}}{\Omega_{1}}=\frac{3 n_{p}}{\omega_{1}} I_{r}^{\prime 2} \frac{R_{r}^{\prime}}{s}=\frac{3 n_{p}}{2 \pi}\left(\frac{E_{s}}{f_{1}}\right)^{2} \frac{s f_{1} R_{r}^{\prime}}{\left(R_{r}^{\prime}\right)^{2}+s^{2} \omega_{1}^{2} L_{r \sigma}^{2}}
$$

The type(10) is strictly constant flux control equation of the mechanical characteristic. If order $d T_{s} / d s=0$, it can get the maximum torque equation as follow as type(11).

$$
T_{e \max }=\frac{3 n_{p}}{8 \pi^{2}}\left(\frac{E_{s}}{f_{1}}\right)^{2} \frac{1}{L_{r \sigma}^{\prime}}
$$

It is easy to know that the maximum torque is constant when $\frac{E_{s}}{f_{1}}=$ const, and it can show the output capacity of motor torque is constant. It can achieve constant flux control when $\frac{E_{s}}{f_{1}}=$ const, but the actual input of motor is stator phase voltage $U s$, and the stator winding EMF is difficult to measure directly, so to achieve precise control is not easy. However, it can get type(12) from the formula $(11)^{[6]}$.

$$
\begin{aligned}
& T_{e}=\frac{P_{e m}}{\Omega_{1}}=\frac{3 n_{p}}{\omega_{1}} I_{r}^{\prime 2} \frac{R_{r}^{\prime}}{s}=\frac{3 n_{p} U_{s}^{2} R_{r}^{\prime} / s}{\omega_{1}\left[\left(R_{s}+\frac{R_{r}^{\prime}}{s}\right)^{2}+\omega_{1}^{2}\left(L_{s \sigma}+L_{r \sigma}^{\prime}\right)^{2}\right]} \\
& =\frac{3 n_{p}}{2 \pi}\left(\frac{U_{s}}{f_{1}}\right)^{2} \frac{s f_{1} R_{r}^{\prime}}{\left(s R_{s}+R_{r}^{\prime}\right)^{2}+s^{2}\left(L_{s \sigma}+L_{r \sigma}^{\prime}\right)^{2}}
\end{aligned}
$$

When the motor operates in the steady state, the speed is close to synchronous speed, and $s$ is very small. So it can be neglected, and the type(13)can be got.

$$
T_{e}=\frac{3 n_{p}}{2 \pi}\left(\frac{U_{s}}{f_{1}}\right)^{2} \frac{s f_{1}}{R_{r}^{\prime}}
$$

From the type(13) it can be seen that the mechanical properties is parallel to descend when we change frequency for the same torque $T e$. It also can achieve very good speed regulator. On the other hand, it can ignore the stator winding and magnetic flux leakage impedance drop when the electromotive force is high from the motor voltage balance equation, so it can be thought $U_{s} \approx E_{s}$.
When the speed is low, the stator resistance $R s$ can't be ignored, $U s$ and $E s$ are very small with low frequency, and the stator impedance drop ratio increases, the stator power supply voltage by the partial pressure of $R s$ for generating magnetic flux of induction electromotive force $E s$ will become smaller, thereby causing the air gap flux weakening the maximum torque $T e_{\max }$ decreases, with a load capacity is reduced. Therefore, in order to improve the low speed performance in low speed on the voltage $U s$ compensation ${ }^{[7]}$.

So it can get the $U / f$ curve from the above theory, then it can get the principle block diagram of constant voltage frequency ratio control system according to the operating frequency and the frequency converter theory to get the block diagram as follow as Figure4.

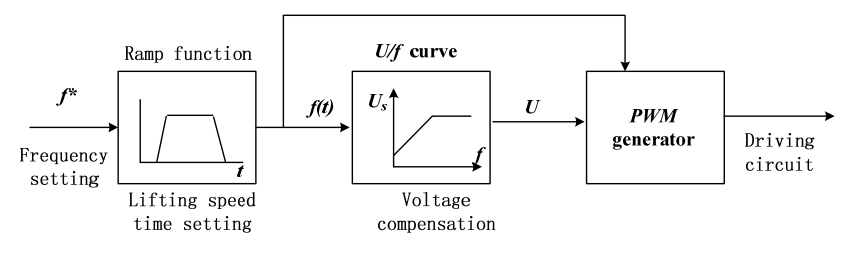

Figure4. The block diagram of variable frequency speed control system

\section{MODELING AND SIMULATION OF ASYNCHRONOUS MOTOR FOR VOLTAGE FREQUENCY RATIO CONTROL SYSTEM BASED ON MATLB}

\section{A. The simulation parameters settings of asynchronous motor}

The motor parameters are as follows: stator winding phase resistance: $R=0.435 \Omega$; rotor winding phase resistance: $R=0.816 \Omega$; phase inductance: $L=0.002 H$; the rotational inertia of the stator and rotor: $J_{s}=J_{r}=1.99 \mathrm{Kgm}^{2}$; motor pole pairs: $P=2$; rated voltage: $380 \mathrm{~V}$.

\section{B. The simulation models of voltage frequency ration control system for asynchronous motor based on Matlab}

The set of work frequency for mathematical model shown in Figure 5 is the set of speed. In order to avoid great current and torque impact caused by the rise of rotational speed rate, the model makes it slow speed fluctuation rate through the ramp function generator, then sent it to $U / f$ generator. According to the frequency corresponding to the determined voltage, the model keeps the $U / f$ generator produces the required stator voltage and frequency, and the given voltage compares with the control signal generated by the PWM generator, then to control inverter, thus realizing the variable frequency speed regulation. 


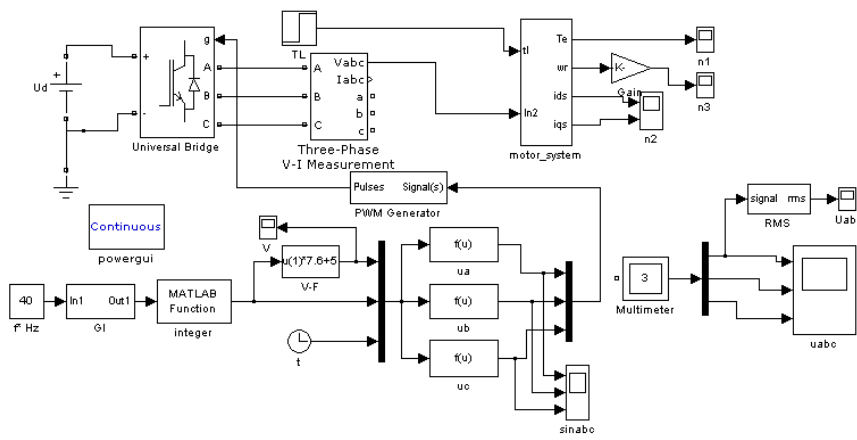

Figure5. The simulation model of voltage frequency ration control system

\section{The simulation results and analysis}

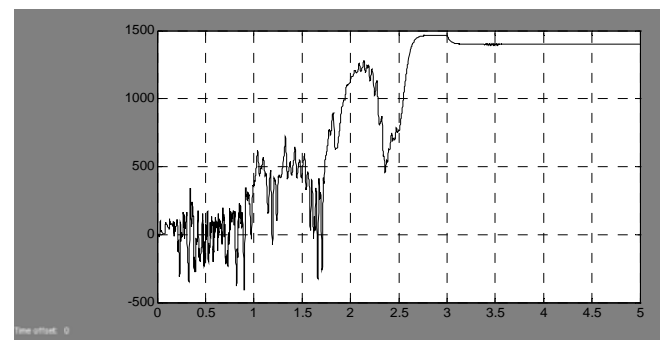

Figure6. The simulation waveform of speed for $50 \mathrm{~Hz}$

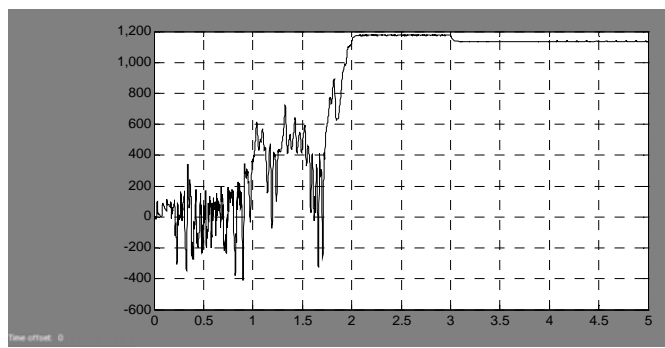

Figure7. The simulation waveform of speed for $40 \mathrm{~Hz}$

The rotation frequency of Figure 6 is $1500 / 50=30$, and the speed frequency ratio of Figure 7 is $1200 / 40=30$. It is easy to see that the speed frequency are equal in two frequency, and the frequency ratio is 30 . Thus the system can adjust the working frequency to achieve the speed smooth regulation. But when the load torque is changed in the third seconds, the speed doesn't recover to the original speed, which shows that the speed tracking of the control system is not good, and it is also the defect for open-loop speed.

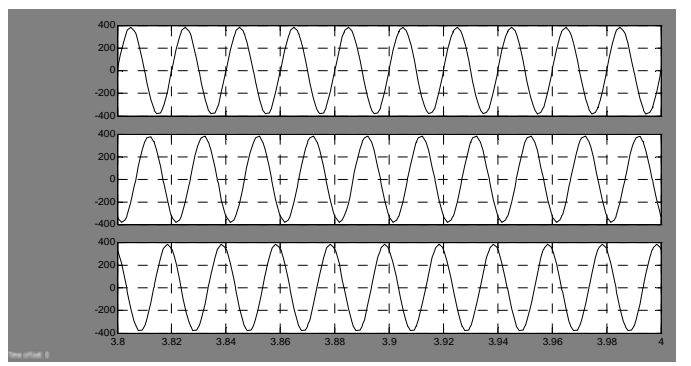

Figure8. The $50 \mathrm{~Hz}$ modulation waveform

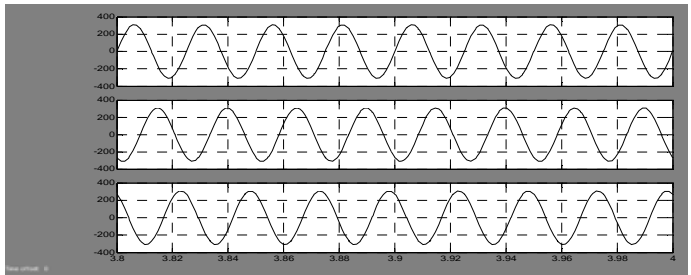

Figure9. The $40 \mathrm{~Hz}$ modulation waveform

From the Figure8 and Figure9, it is easy to see the frequency of modulated wave is $50 \mathrm{~Hz}$ and amplitude modulation wave is 380 of the rated value when the work frequency is $50 \mathrm{~Hz}$ from the Figure10. At the same time, the frequency of modulated wave is $40 \mathrm{~Hz}$ and amplitude modulation wave is 300 of the rated value when the work frequency is $40 \mathrm{~Hz}$ from the Figure 11 . Thus changing the input frequency can change the inverter output voltage amplitude and frequency, and it is to say that it can change the input of asynchronous motor. So it has been validated by changing the frequency to control asynchronous motor through above principle.

\section{CONCLUSION}

According to working principle of the asynchronous motor, the paper deduces the mathematical model, and builds the simulation model of $V / f$ speed-adjusting control system based on Matlab/Simulink. At last the paper gives the simulation results and analyzes the results. The simulation results demonstrate the validity of the theoretical analysis, which provides a theoretical basis for the development for asynchronous motor.

\section{REFERENCES}

[1] Ma Xiaoliang. High-performance VVVF and typical control system[M]. Beijing: China Machine Press, 2001 (In Chinese)

[2] Chen Boshi. Electric drive control system - motion control systems. 3rd ed. Beijing: China Machine Press, 2003 (In Chinese)

[3] Blaschke F. The principle of Field-orientation as Applied to the New Transvektor Closed-loop Control System for Rotating-Field Machine [J]. Stemens Review, 1972,34:217-220.

[4] Depenbrock M. Direct Self Control(DSC) of Inverter-fed Induction Machines[J].IEEE Trans.PE,1988,3(4):420-429.

[5] Ji Zhi-Cheng, ShenYan-Xia, Jiang Jianguo. "the new modeling and simulation method of BLDCM system Based on Matlab."System Simulation, 2003,12. Pages:1745-1749 (In Chinese)

[6] Tae-Hyung Kim, Byung-Kuk Lee, Ehsani, M., Sensorless control of the BLDC motors from near zero to high speed, Applied Power Electronics Conference and Exposition[J], 2003. APEC , 03. Eighteenth Annual IEEE, Volume: 1, 9-13 Feb. 2003, Pages:306-312 vol.1

[7] A. MILOUDI, Eid A. AL-RADADI, A. D. DRAOU. A Variable Gain PI Controller Used for Speed Control of a Direct Torque Neuro Fuzzy Controlled Induction Machine Drive[J]. Turk J Elec Engin,2007,(15):3749.

[8] Hong Naigang. The MATLAB Simulation of power electronics and electric drive control system. Beijing: China Machine Press, 2006 (In Chinese 